\title{
CARACTERIZACIÓN DE IGM, IGG TOTAL, IGG1 Y ANTICUERPOS DE CADENA PESADA EN CALOSTRO DE LLAMAS (LAMA GLAMA) MEDIANTE ELISA
}

\section{CHARACTERIZATION OF IGM, TOTAL IGG, IGG1 AND HCABS IN COLOSTRA OF \\ LLAMAS (LAMA GLAMA) BY ELISA}

Nicolás Caggiano ${ }^{1}$, Natalia Saccodossi ${ }^{2}$, Teresa Gentile ${ }^{2}$, María Angelina Chiappe Barbará ${ }^{1}$, Juliana Leoni ${ }^{2}$ y Emilio De Simone ${ }^{1}$

1 Cátedra de Fisiología Animal, Facultad de Ciencias Veterinarias.

2 Cátedra de Inmunología Facultad de Farmacia y Bioquímica. Universidad de Buenos Aires. República Argentina

\section{RESUMEN}

Objetivos: determinar los niveles de IgM, IgG total y Anticuerpos de Cadena Pesada (HCAbs; por su sigla en inglés Heavy Chain Antibodies) (IgG2 e IgG3) en calostro de llamas y evaluar la concentración de HCAbs en relación a la IgG total y al isotipo convencional IgG1.

Métodos: en este estudio se utilizaron 15 llamas preñadas, que fueron ordeñadas dentro de las primeras 24 horas post-parto. Se diseñaron ELISAs Sandwich para la cuantificación de IgM total, IgG total e IgG1. La concentración de HCAbs fue calculada mediante la diferencia entre IgG total e IgG1. (HCAbs =IgG total-IgG1).

Resultados: los niveles encontrados fueron: $\operatorname{IgM}=17.02 \mathrm{mg} / \mathrm{ml}$ (DS=9.85) $\mathrm{IgG}$ total= 42.54 $\mathrm{mg} / \mathrm{ml}(\mathrm{DS}=27.79), \operatorname{IgG1}=24.34 \mathrm{mg} / \mathrm{ml}(\mathrm{DS}=13.96)$ y HCAbs $(\mathrm{IgG}$ total- $\mathrm{IgG} 1)=18.19 \mathrm{mg} / \mathrm{ml}$ (DS=15.49). Los resultados de $\mathrm{IgG}$ fueron consistentes en relación a lo descripto en otras especies con similar tipo de placentación. Los HCAbs representan el 43\% de IgG calostral y la concentración de IgM presentó valores muy altos (dos y tres veces más elevados) que los descriptos en las especies con similar tipo de placentación. 
Conclusión: este estudio es el primero que evalúa la concentración de cada isotipo de inmunoglobulinas en calostro de llamas. Describimos niveles de IgM más elevados en comparación con especies con similar placentación. El isotipo IgG1, que representa el isotipo convencional, es el anticuerpo más abundante en calostro, siendo los HCAbs la segunda fracción más importante. Esta información puede ser útil en el manejo de neonatos con fallas en la transferencia de inmunidad pasiva mediante el calostro.

Palabras claves: Calostro, llama, HCAbs, IgM.

\section{ABSTRACT}

Objectives: to determine the levels of IgM, total IgG, IgG1 and HCAbs (IgG2 and IgG3) in colostra of llamas and to evaluate the concentration of HCAbs in relation to total IgG and the conventional isotype IgG1.

Methods: Fifteen pregnant llamas were used in this study, which were milked within the first 24 hours after delivery. Sandwich ELISAs were designed for the quantification of IgM, total IgG and IgG1. The HCAbs concentration was calculated by difference between total IgG and IgG1 $(\mathrm{HCAbs}=$ total $\mathrm{IgG}-\mathrm{IgG} 1)$.

Results: The levels of Igs were found to be: $\operatorname{IgM}=17.02 \mathrm{mg} / \mathrm{ml}(\mathrm{SD}=9.85)$, total $\operatorname{IgG}=$ $42.54 \mathrm{mg} / \mathrm{ml}(\mathrm{SD}=27.79), \mathrm{IgG} 1=24.34 \mathrm{mg} / \mathrm{ml}(\mathrm{SD}=13.96)$ and HCAbs $(\operatorname{IgGtotal}-\mathrm{IgG} 1)=18.19$ $\mathrm{mg} / \mathrm{ml}(\mathrm{SD}=15.49)$. Results regarding total $\mathrm{IgG}$ are consistent with those described in other species with similar type of placentation. HCAbs represented $43 \%$ of colostral $\operatorname{IgG}$ and $\operatorname{IgM}$ concentration presented values that were much higher (two and three times respectively) than those described for species with similar type of placentation.

Conclusions: This study is the first which evaluate amounts of each immunoglobulin isotype in colostra of llamas. We described a higher value of $\operatorname{IgM}$ in in comparison with species with similar placentation type. The IgG1 isotype, which represents the conventional isotype, is the more abundant antibody in colostra, being the HCAbs the second important fraction. This information may be useful in the management of neonates with failures in the transference of maternal passive immunity by colostra.

Keywords: Colostrum, llama, HCAbs, IgM. 


\section{INTRODUCCIÓN}

Los Camélidos Sudamericanos (CS) incluyen llamas, alpacas, guanacos y vicuñas. Estudios genéticos demostraron que la alpaca y la vicuña son muy similares, mientras que el ancestro de la llama es el guanaco (Kadwell et al., 2001). Durante siglos la llama y la alpaca han sido domesticadas, no ocurrió lo mismo con el guanaco y la vicuña que son especies silvestres. Los CS fueron arrastrados a hábitats marginales por presiones de caza y por la cría extensiva de ganado. A pesar de lo anteriormente dicho, los CS muestran una gran capacidad de adaptación a ambientes considerados extremos para la ganadería tradicional. En América del Sur estos animales son explotados a alturas de 3.000 metros sobre el nivel del mar. La producción de CS se ha vuelto importante para la industria agrícola-ganadera principalmente por productos como la fibra, la carne y el cuero (Lichtenstein y Vilá, 2003).

El control sanitario es una de las piedras angulares en la optimización de la producción ganadera. Aunque hay varias vacunas que son comúnmente usadas en CS, estas vacunas fueron originalmente desarrolladas para otras especies de ganado. Una efectiva implementación de planes de vacunación y de estudios en la seroprevalencia de enfermedades infecciosas que afecten a los CS requiere una mayor comprensión de sistema inmune de dichos camélidos. El status inmunológico de un neonato es altamente dependiente de las inmunoglobulinas (Igs) transferidas por calostro.

Así como en los bovinos, equinos y ovinos, los CS tienen un tipo de placenta en la cual la transmisión materno-fetal de Igs durante la gestación no es posible (Fowler y Olander, 1990). Por esta razón, la única fuente de IgG materna es el calostro. Como en otras especies relacionadas, el epitelio intestinal de los camélidos permite la absorción de grandes moléculas como Igs durante las primeras 24 horas de vida.

Los CS tienen una pobre eficiencia reproductiva comparada con otras especies. Uno de los puntos principales en la producción de estas especies es el alto nivel de mortalidad durante el período perinatal, siendo uno de los períodos más vulnerables para la supervivencia. La mortalidad neonatal está relacionada con la falla en la transferencia de inmunidad pasiva de Igs que ocurre en aproximadamente en un 9\% de las alpacas en Perú según Garmendia A y McGuire T (1987), o un 20\% de las llamas y alpacas en EE.UU según Weaver D et al. (2000). La carencia de inmunidad adquirida aumenta la susceptibilidad a enfermedades y la tasa de mortalidad de camélidos, lo cual representa serios problemas económicos.

Al nacimiento, las llamas y alpacas recién nacidas son hipogammaglobulinémicas, teniendo valores de IgG en plasma indetectables (Weaver et al., 2000; Bravo et al., 1997). La 
concentración sérica de $\mathrm{IgG}$ al nacimiento no fue precisamente determinada, aunque algunos

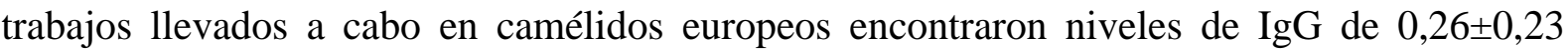
$\mathrm{mg} / \mathrm{ml}$, (Wernery, 2001). Esta concentración es marcadamente más baja que en una llama adulta $(8,93 \pm 1,815 \mathrm{mg} / \mathrm{dl})$, (De Simone et al., 2008). A las 48 horas de ingerido el calostro la concentración de IgG es de 23,29 mg/ml (Bravo et al., 1997).

Garmendia et al. (1987) consideraron que la transferencia de IgG ocurre cuando la IgG sérica de los neonatos es cercana a los $9 \mathrm{mg} / \mathrm{ml}$ a las 48 horas posteriores a la ingesta de calostro. El pico de concentración de Igs séricas luego de la ingesta de calostro se da entre el primer y el segundo día de vida (Bravo et al, 1997). Vale la pena mencionar que la vida media de la IgG calostral en plasma de una llama recién nacida es de 15,7 días, siendo este valor similar para las alpacas (Weaver et al., 2000). Por otro lado, la concentración en plasma de HCAbs fue demostrado que declina más rápidamente que la $\operatorname{IgG}$ convencional (50\% de reducción en 14 días vs. 20 a 25 días respectivamente), (Daley-Bauer et al., 2010). Bravo et al, vieron en 1997 que la concentración de $\operatorname{IgG}$ en la glándula mamaria de llamas y alpacas previo al parto alcanzaba valores máximos que sobrepasaba en diez veces los valores séricos y que la cantidad de proteína declinaba rápidamente luego del primer día post parto. Una inadecuada nutrición durante la gestación tardía puede reducir la cantidad y la calidad de calostro.

En 1993, Hamers-Castermans et al., describieron, en suero de camélidos, la presencia de isotipos de IgG a los cuales les faltaban cadenas livianas. Este tipo de Igs han sido llamadas Anticuerpos de Cadena Pesada. En la familia Camelidae, al menos tres subclases de IgG fueron descriptas (IgG1, IgG2 e IgG3), de los cuales la IgG2 y la IgG3 son HCAbs, mientras que la IgG1 tiene la estructura convencional. Diferentes subisotipos han sido clasificados mediante análisis genómicos de secuencias de cadenas de ADN correspondientes a la región bisagra, IgG1 tiene dos subisotipos (IgG1a e IgG1b) y la IgG2 tiene tres (IgG2a, IgG2b e IgG2c) (Nguyen et al., 1993; Vu et al., 1997). Estructuralmente los HCAbs carecen del dominio CH1, aunque las secuencias codificadoras están presentes. La ausencia del dominio CH1 en la molécula de Ig es a causa de una remoción post-transcripcional al exón correspondiente (Nguyen et al. 1998; De Genst et al,. 2006).

Se cree que la presencia de los HCAbs podría ayudar a los camélidos a desarrollar una respuesta inmune humoral contra microorganismos que modifican sus antígenos de superficie como mecanismo de escape (Stijlemans et al., 2004). La distribución y las características del tejido linfoideo, el proceso de cambio de isotipo y la relación entre isotipos y la respuesta TH1 y TH2 de los camélidos no han sido estudiadas detalladamente. Además, el rol biológico de los 
HCAbs en las especies que los producen no ha sido determinado, así como su concentración y su rol biológico en la inmunidad pasiva.

En un trabajo previo hemos desarrollado un anti-suero contra isotipos de llama que nos permitió medir IgM y las distintas las subclases de IgG mediante ELISA (De Simone et al., 2008; Saccodossi et al., 2006). En este trabajo, realizamos modificaciones en el ELISA anteriormente citado para la cuantificación de $\operatorname{IgG}$ total, IgM e IgG1 en calostro. Es digno de destacar que los trabajos realizados por otros autores se centraron en la medición de inmunoglobulina total solamente por métodos semicuantitativos.

El objetivo de este trabajo es determinar los niveles de IgM, IgG total, IgG1 y HCAbs (IgG2 e IgG3) en calostro de llamas y evaluar la concentración de HCAbs en relación a IgG total y el isotipo convencional IgG1.

\section{MATERIALES Y MÉTODOS}

\section{Animales y muestras}

En este estudio se utilizaron 15 llamas preñadas, las cuales fueron ordeñadas dentro de las primeras 24 horas post-parto. Por cada llama se tomó una muestra de $15 \mathrm{ml}$ de calostro el cual fue obtenido mediante ordeñe manual. Los animales muestreados pertenecen a un establecimiento que se dedica a la cría de llamas ubicado en la provincia de San Luis, Argentina. Todas las madres gozaban de buena salud y de un estado nutricional adecuado. Las muestras una vez obtenidas fueron mantenidas a $-20^{\circ} \mathrm{C}$ hasta su utilización.

\section{Anticuerpos}

Sueros policlonales de anti-IgM, anti-IgG total y anti-IgG1 (dominio CH1) fueron obtenidos mediante la inmunización de conejos como fue previamente descripto (De Simone et al., 2008). También se utilizó un anti-IgG de llama (H+L) policlonal de cabra conjugado con una peroxidasa (HRP; Bethyl Laboratories Inc., Montgomery, Tex.).

\section{ELISAs Sandwich}

Los ELISAs Sándwich fueron diseñados para la cuantificación de IgM, IgG total e IgG1. La concentración de los HCAbs fue calculada mediante la diferencia entre IgG e IgG1 (HCAbs= IgG total- IgG1). 
Para la determinación de la IgG total, se utilizó una placa de 96 micropocillos (Nunc, Dinamarca) los cuales fueron cubiertos con $10 \mu \mathrm{g}$ de anti-IgG de llama hecho en conejo' (De Simone E et al., 2008), e incubado durante 1 hora a $37^{\circ} \mathrm{C}$. Los pocillos fueron lavados con una solución salina de fosfato bufferada con un 0,05\% de Tween 20 (PBST; del inglés phosphatebuffered saline Tween) y bloqueada con un 3\% de leche descremada en PBS durante la noche a $4^{\circ} \mathrm{C}$. Las muestras de calostro fueron agregadas en una dilución de 1:100.000 en una solución de PBS con un $3 \%$ de leche descremada e incubadas durante 1 hora a $37^{\circ} \mathrm{C}$. Los pocillos fueron lavados cinco veces con PBST e incubados con el anti-IgG de llama conjugado con HRP, realizado en conejo, diluido 1:2.000 en una solución de PBS con un 3\% de leche descremada e incubados durante 1 hora a $37^{\circ} \mathrm{C}$. Los pocillos fueron lavados de la misma manera que anteriormente y la reacción colorimétrica se llevó a cabo mediante una solución de $\mathrm{H}_{2} \mathrm{O}_{2}$-TMB. Una curva patrón fue realizada con IgG purificada en un rango de 31,25 ng/ml a $100 \mathrm{ng} / \mathrm{ml}$ mediante diluciones al medio. La densidad óptica fue medida mediante un lector de placas Rayto RT-2100C a $450 \mathrm{~nm}$.

El ELISA sándwich para la determinación de IgM total fue realizado de la siguiente manera: los pocillos fueron cubiertos con $10 \mu \mathrm{g}$ de anti-IgM de llama realizado en conejo como se describió previamente. Luego del lavado y el bloqueado, como se indicó anteriormente, las muestras de calostro fueron agregadas en una dilución de 1:100 e incubadas durante 1 hora a $37^{\circ} \mathrm{C}$. Luego de cinco lavados, los pocillos fueron incubados con un anti-IgM de llama conjugado con HRP realizado en conejo en una dilución de 1:200. Este anticuerpo secundario fue marcado como se describió previamente, (De Simone et al., 2008). Luego de incubar y lavar, las reacciones se realizaron como se describió previamente. Una curva patrón fue diseñada con IgM, (De Simone et., 2006), purificada en un rango de 0,15 $\mu \mathrm{g} / \mathrm{ml}$ a $100 \mu \mathrm{g} / \mathrm{ml}$ mediante diluciones al medio.

El ELISA sándwich para determinar IgG1 total fue preparado de manera similar. Primero $10 \mu \mathrm{g}$ de anti-IgG1 de llama fue cargado en cada pocillo e incubado durante 1 hora a $37^{\circ} \mathrm{C}$. Luego la placa se incubó durante toda la noche a $5^{\circ} \mathrm{C}$ con leche descremada al $3 \%$ en una solución de PBS. A continuación las muestras de calostro fueron incubadas en una dilución de 1:200 en una solución de PBS con un $3 \%$ de leche descremada durante 1 hora a $37^{\circ} \mathrm{C}$. Finalmente los pocillos fueron incubados con el anti-IgG de llama conjugado con HRP sintetizado en conejo en una dilución de 1:2000. La reacción colorimétrica se desarrolló con la adición de TMB. La curva patrón fue preparada con $\operatorname{IgG} 1$ purificada a una concentración de $100 \mu \mathrm{g} / \mathrm{ml}$ a $0,15 \mu \mathrm{g} / \mathrm{ml}$. La densidad óptica fue leída mediante un lector de placas de ELISA a $450 \mathrm{~nm}$. 
Los HCAbs fueron determinados mediante la diferencia entre IgG total e IgG1.

\section{Análisis estadísticos}

Los análisis estadísticos y los gráficos fueron realizados mediante el programa Graph pad prism 7.0.

\section{RESULTADOS}

La tabla y la figura 1 muestran los valores (media y desvío estándar) y el rango para IgM, IgG total, IgG1 y HCAbs de las muestras de calostro. Igualmente observaciones previas en terneros (Shearer et al., 1992) dieron como resultado que un 20\% (3/15) de las muestras tuvieron baja concentración de $\operatorname{IgG}(<20 \mathrm{mg} / \mathrm{ml})$, un $40 \%$ (6/15) tuvieron una concentración de IgG intermedia (entre 20-50 mg/ml) y el 40\% (6/15) restante tuvo una concentración de IgG calostral alta (>50 mg/ml, Figure 2).

\begin{tabular}{c|cccc} 
& IgM & IgG total & IgG1 & HCAbs \\
& & & & \\
\hline & & & & \\
Media & 17.03 & 42.55 & 24.35 & 18.19 \\
\hline DS & 9.859 & 27.79 & 13.97 & 15.50 \\
\hline & & & & \\
\hline & & & & \\
Rango & $2.82-37.26$ & $7.46-90.36$ & $4.6-52.02$ & $1.6-47.96$
\end{tabular}

Tabla 1. Media (mg/ml), Desvío Standard (SD) y rango para cada inmunoglobulina calostral. 
La figura 1 muestra una gran variación en la concentración de $\operatorname{IgG}$ entre diferentes muestras de calostro. La concentración de IgG en el calostro de llamas, determinada mediante el método de ELISA, fue parecida a otras especies con un tipo de placentación similar. Sin embargo, en este trabajo, nos enfocamos en la evaluación de la cantidad de IgG1 y HCAbs. El isotipo $\mathrm{IgG} 1$ representa el $57.2 \%$ del total de $\mathrm{IgG}$, siendo este porcentaje levemente menor que el encontrado en suero de llamas. En 11/15 de las muestras e observó que el mayor isotipo de IgG encontrado fue IgG1, mientras que en 4/15 de las muestras la fracción de IgG predominante fue la de HCAbs.
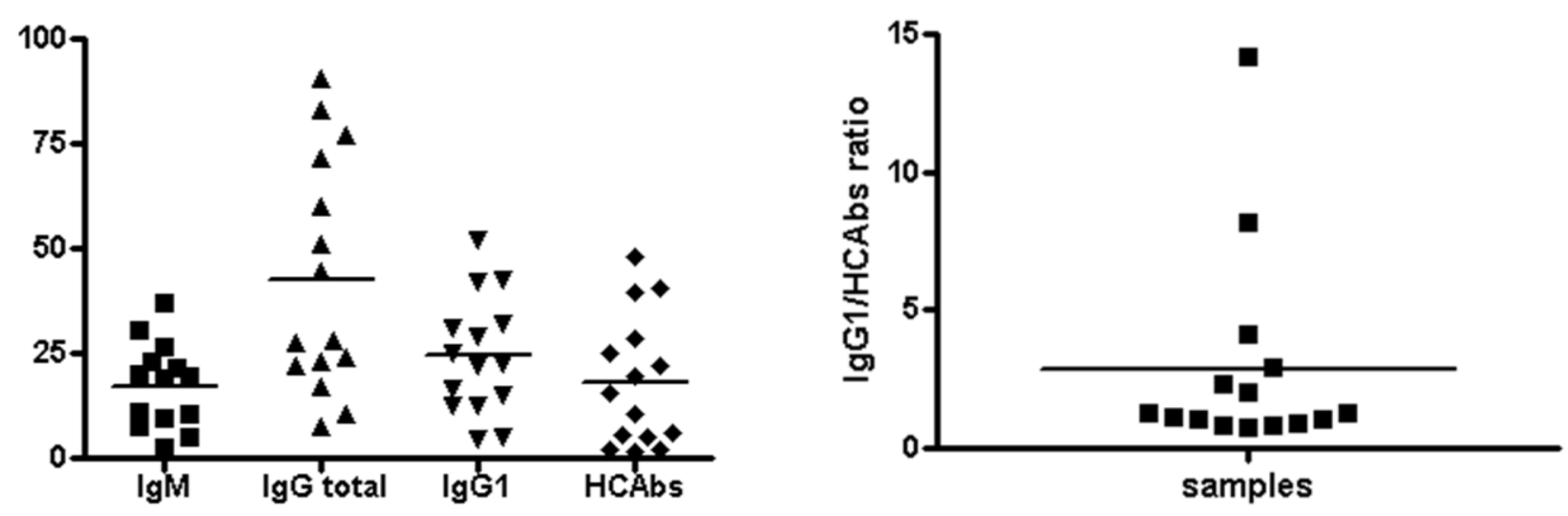

Figura 1. Valores de en muestras de calostro. Figura 3. Valores de relación IgG1/HCAbs en muestras de calostro.

También se calculó la relación IgG1/HCAbs y se observaron valores que rondaban a 1 $(0,74-2,94)$ en $12 / 15$. Los valores más altos $(>4)$ hallados en el resto de las muestras fue debido a la baja concentración de HCAbs de las muestras (Figura 3).

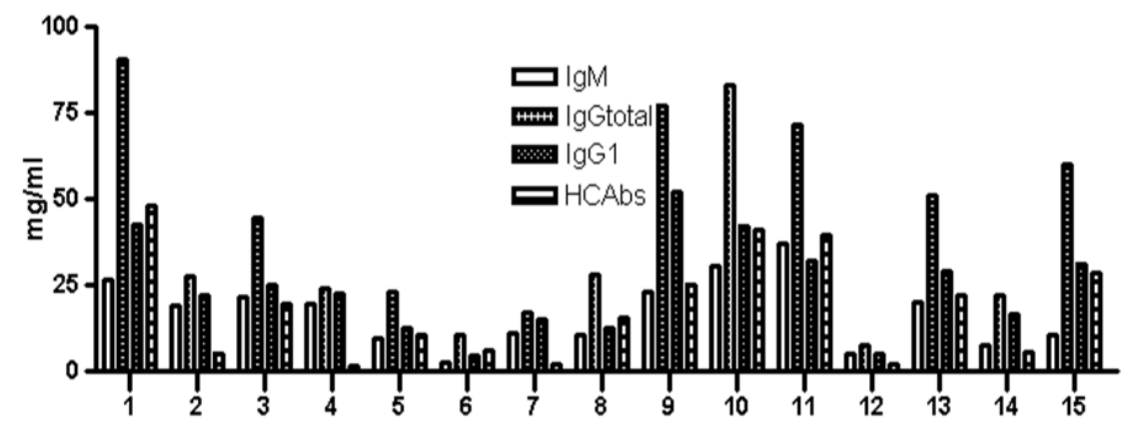

Figura 2. Valores de IgM, IgG total, IgG1 y HCAbs en cada muestra de calostro. 


\section{DISCUSIÓN}

Los resultados obtenidos fueron similares a los obtenidos en aquellas especies con similar tipo de placentación. Trabajos realizados en bovinos demostraron que la concentración de Igs en calostro fue de entre 32 a $212 \mathrm{mg} / \mathrm{ml}$ con un valor promedio de $8,7 \mathrm{mg} / \mathrm{ml}$ de $\mathrm{IgG}$ e IgM respectivamente'(Hurley, 2003). En bovinos el isotipo mayormente encontrado en calostro es el IgG1, con valores de concentración que van de 9 a 166 mg/ml' (Baumrucker et al., 2010). Valores similares también fueron encontrados por otros autores, (Davis y Drackley, 1998; Murphy et al., 2005). En cabras la inmunoglobulina mayoritaria es la IgG con valores de 59,9 $\mathrm{mg} / \mathrm{ml}$, mientras que el promedio de IgM fue de 6,14 mg/ml, (Chen et al., 2008). En la especie anteriormente dicha, Yang et al. (2009) encontraron concentraciones de IgG de 72,01 mg/ml. En equinos la IgG es también la Ig mayoritaria con valores que van en un rango entre 31 y 60 mg/ml, (Rouse e Ingram, 1970).

En este trabajo los valores de $\operatorname{IgM}$ encontrados fueron considerablemente altos comparados con otras especies con similar placentación. El último hallazgo podría indicar la existencia de un papel especial por parte de la $\operatorname{IgM}$, por ejemplo un efecto protector en el tracto digestivo del recién nacido. La concentración de IgM encontradas en nuestras muestras fue dos veces más alta que las encontradas en otras especies. Por ejemplo, en calostro de bovinos los valores hallados por otro autor fueron de entre 5,8 y 13,4 mg/ml, (Porter, 1972), con un valor promedio de 3,19 mg/ml (Rudovsky et al., 2008), mientras que en porcinos los valores promedio de IgM fueron de 3,2 mg/ml, (Bourne, 1973).

\section{CONCLUSIÓN}

Muchos autores han descripto que los dominios VHH de los HCAbs tienen importantes ventajas en comparación con las Igs convencionales, sin embargo las propiedades fisiológicas de la molécula de HCAbs en su totalidad no parece tener alguna ventaja cuando se compara con las Igs convencionales, (Ferrari et al., 2007). Además nosotros no fuimos capaces de demostrar funciones efectoras como fijación de complemento por parte de los HCAbs, cuando las Igs convencionales tienen sitios de fijación para C1q, (Saccodossi et al., 2012). Otro estudio demostró que los HCAbs no aparecen en las respuestas inmunes de manera temprana sino luego de la acción de IgG1 (De Simone et al., 2008). En este estudio demostramos la presencia de HCAbs en calostro, sin embargo el isotipo predominante fue la molécula convencional de IgG. Este trabajo nos acerca al esclarecimiento del rol de los HCAbs en la inmunidad pasiva. 
Estudios futuros son necesarios para mejorar estrategias profilácticas basadas en la vacunación y en la transferencia de Igs a los neonatos.

\section{Agradecimientos}

Los autores agradecen el soporte financiero de la Universidad de Buenos Aires, Proyecto No. UBACyT 20020100200080.

\section{BIBLIOGRAFIA}

Baumrucker CR, Burkett AM, Magliaro-Macrina AL, Dechow CD.2010. Colostrogenesis: mass transfer of immunoglobulin G1 into colostrum. J Dairy Sci 93:3031-3038.

Bourne FJ. The immunoglobulin system of the suckling pig. 1973. Proc Nutr Sac 32:205-215.

Bravo PW, Garnica J, Fowler ME. 1997. Immunoglobulin G concentrations in periparturient llamas, alpacas and their crias. Small Rumin Res 26:145-149.

Chen S, Zhao S, Shi B, Zeng S, Ren F. 2008. Study on Changes of Composition and Immunoglobulin Constituents of Goat Colostrum. Food Science 29:41-44.

Daley-Bauer LP, Purdy SR, Smith MC, Gagliardo LF, Davis WC, Appleton JA. 2010. Contributions of Conventional and Heavy-Chain IgG to Immunity in Fetal, Neonatal, and Adult Alpacas. Clin Vaccine Immunol 17:2007-2015.

Davis CL, Drackley JK. 1998. The development, nutrition, and management of the young calf. Iowa State University Press, Ames, Iowa.

De Genst E, Saerens D, Muyldermans S, Conrath K. 2006. Antibody repertoire development in camelids. Dev Comp Immunol 30:187-198.

De Simone E, Saccodossi N, Ferrari A, Leoni L, Leoni J. 2006. Immunochemical analysis of IgG subclasses and IgM in South American camelids. Small Rumin Res 64: 2-9.

De Simone EA, Saccodossi N, Ferrari A, Leoni J. 2008. Development of ELISAs for the measurement of IgM and $\operatorname{IgG}$ subclasses in sera from llamas (Lama glama) and assessment of the humoral immune response against different antigens. Vet Immunol and Immunopathol 126:64-73.

Ferrari A, Rodríguez MM, Power P, Weill FS, De Simone EA, Gutkind G, Leoni J. 2007. Immunobiological role of llama heavy-chain antibodies against a bacterial betalactamase. Vet Immunol Immunopathol 15:173-182.

Fowler ME, Olander HJ. 1990. Fetal membranes and ancillary structures of llamas (Lama glama). Am J Vet Res 51:1495-1500. 
Garmendia A, McGuire T. 1987. Mechanism and isotypes involved in passive immunoglobulin transfer to the newborn alpaca (Lama pacos). Am J Vet Res 48:1465-1471.

Hamers-Casterman C, Atarhouch T, Muyldermans S, Robinson G, Hamers C, Songa EB, Bendahman N, Hamers R. 1993. Naturally occurring antibodies devoid of light chain. Nature 363:446-448.

Hurley WL. 2003. Immunoglobulins in mammary secretions. In: Fox, P.F., McSweeney, P.L.H.

(Eds.), Advanced Dairy Chemistry. Volume 1. Proteins. Part A, Kluwer Academic/Plenum Publishers, New York 422-447.

1. Kadwell M, Fernandez M, Stanley HF, Baldi R, Wheeler JC, Rosadio R, Bruford MW. 2001. Genetic analysis reveals the wild ancestors of the llama and the alpaca. Proc R Soc Lond B 268:2575-2584

Lichtenstein G, Vilá B. 2003. Vicuna Use by Andean Communities: An Overview. Mountain Res Dev 23:197-201.

Murphy BM, Drennan MJ, O’Mara FP, Earley B. 2005. Cow serum and colostrum immunoglobulin (IgG1) concentration of five suckler cow breed types and subsequent immune status of their calves. Ir J Agr Food Res 44:205-213.

Nguyen VK, Muyldermans S, Hamers R. 1998. The specific variable domain of camel heavychain antibodies is encoded in the germline. J Mol Biol 275:413-418.

Nguyen VK, Desmyter A, Muyldermans S. 2001. Functional Heavy-Chain Antibodies in Camelidae. Adv Immunol 79:261-296.

Porter P. 1972. Immunoglobulins in Bovine Mammary Secretions, quantitative changes in early lactation and absorption by the neonatal calf. Immunology 23:225-238.

Rouse BT, Ingram DG. 1970. The Total Protein and Immunoglobulin Profile of Equine Colostrum and Milk. Immunology. 19:901-907.

Rudovsky A, Locher L, Zeynerc A, Sobiraj A, Wittek T. 2008. Measurement of immunoglobulin concentration in goat colostrums. Small Rumin Res 74:265-269.

Saccodossi N, De Simone E, Leoni J. 2006. A novel and easy method for the production of recombinant peptides for use in the generation of monospecific antisera against Lama glama IgG2b and IgG2c subclasses. J Immunol Methods 313:214-218.

Saccodossi N, De Simone EA, Leoni J. 2012. Structural analysis of effector functions related motifs, complement activation and hemagglutinating activities in Lama glama heavy chain antibodies. Vet Immunol Immunopathol. 145:323-331.

Stijlemans B, Conrath K, Cortez-Retamozo V, Van Xong H, Wyns L, Senter P, Revets H, De Baetselier P, Muyldermans S, Magez S. 2004. Efficient targeting of conserved cryptic 
epitopes of infectious agents by single domain antibodies. African trypanosomes as paradigm. J Biol Chem 279:1256-1261.

Vu KB, Ghahroudi MA, Wyns L, Muyldermans S. 1997. Comparison of llama VH sequences from conventional and heavy chain antibodies. Mol Immunol 34:1121-1131.

Weaver DM, Tyler JW, Scott MA, Wallace LM, Marion RS, Holle JM. 2000. Passive transfer of colostral immunoglobulin $\mathrm{G}$ in neonatal llamas and alpacas. Am J Vet Res 61: 738741.

Weaver DM, Tyler JW, Marion RS, Wallace LM, Nagy JK, Holle JM. 2000. Evaluation of assays for determination of passive transfer status in neonatal llamas and alpacas. J Am Vet Med Assoc 216:559-563.

Wernery U. 2001 Camelid immunoglobulin and their importance for the New-Born. J Vet Med 48:561-568.

Yang XY, Chen JP, Zhang FX. 2009. Research on the chemical composition of Saanen goat colostrums. J Dairy Technology 62:500-504. 